\title{
Suprachiasmatic Modulation of Noradrenaline Release in the Ventrolateral Preoptic Nucleus
}

\author{
Benoît Saint-Mleux, ${ }^{1}$ Laurence Bayer, ${ }^{1}$ Emmanuel Eggermann, ${ }^{1}$ Barbara E. Jones, ${ }^{2}$ Michel Mühlethaler, ${ }^{1}$ and \\ Mauro Serafin ${ }^{1}$ \\ ${ }^{1}$ Département de Neurosciences Fondamentales, Centre Médical Universitaire, 1211 Genève 4, Switzerland, and ²Department of Neurology and \\ Neurosurgery, McGill University, Montreal Neurological Institute, Montreal, Quebec, Canada H3A 2B4
}

As the major brain circadian pacemaker, the suprachiasmatic nucleus (SCN) is known to influence the timing of sleep and waking. We thus investigated here the effect of SCN stimulation on neurons of the ventrolateral preoptic nucleus (VLPO) thought to be involved in promoting sleep. Using an acute in vitro preparation of the rat anterior hypothalamus/preoptic area, we found that whereas single-pulse stimulations of the SCN evoked standard fast ionotropic IPSPs and EPSPs, train stimulations unexpectedly evoked a long-lasting inhibition (LLI). Such LLIs could also be evoked in VLPO neurons by pressure application of NMDA within the SCN, indicating the specific activation of SCN neurons. This LLI was shown to result from the presynaptic facilitation of noradrenaline release, because it was suppressed in presence of yohimbine, a selective antagonist of $\alpha_{2}$-adrenoreceptors. The LLI depended on the opening of a potassium conductance, because it was annulled at $E_{\mathrm{K}}$ and could be reversed below $E_{\mathrm{K}}$. These results show that the SCN can provide an LLI of the sleep-promoting VLPO neurons that could play a role in the circadian organization of the sleep-waking cycle.

Key words: arousal; circadian; noradrenaline; presynaptic mechanism; rat; sleep; wakefulness

\section{Introduction}

The ventrolateral preoptic nucleus (VLPO) has been suggested in recent years to play a major role in promoting sleep (for review, see Saper et al., 2005). Indeed, sleep-promoting neurons of the VLPO appear to be GABAergic (Gallopin et al., 2000; Gong et al., 2004; Modirrousta et al., 2004) and to increase their activity during sleep (Szymusiak et al., 1998) such as to inhibit the major brainstem arousal systems to which they project (Jones, 2005; Saper et al., 2005). In previous studies, we identified and characterized VLPO sleep-promoting neurons in vitro (Gallopin et al., 2000; Saint-Mleux et al., 2004) and demonstrated that they are inhibited by the transmitters of the major arousal systems. Such an inhibition, which would occur when the arousal systems are active, is thought to contribute to the initiation, maintenance, and enhancement of waking.

It has recently been demonstrated that the VLPO receives an input either directly or indirectly from the suprachiasmatic nucleus (SCN) (Novak and Nunez, 2000; Chou et al., 2002; Deurveilher et al., 2002). Given the prime importance of the SCN as the central circadian pacemaker (for review, see Kalsbeek and Buijs, 2002; Mistlberger, 2005), its input to the VLPO could directly affect the circadian organization of the sleep-waking cycle and thus represents an important pathway to study functionally. To explore the relation between the SCN and the VLPO, we de-

\footnotetext{
Received Aug. 7, 2006; revised May 8, 2007; accepted May 8, 2007.

This work was supported by grants from the Swiss Fonds National (M.S.), the Novartis, Ott, de Reuter, and Schmidheiny Foundations (M.M., M.S.), and the Canadian Institutes of Health Research (B.E.J.).

Correspondence should be addressed to Dr. Mauro Serafin, Département de Neurosciences Fondamentales, Centre Médical Universitaire, 1 Rue Michel-Servet, 1211 Genève 4, Switzerland. E-mail: mauro.serafin@ medecine.unige.ch.

DOI:10.1523/JNEUROSCI.1432-07.2007

Copyright $\odot 2007$ Society for Neuroscience $\quad$ 0270-6474/07/276412-05\$15.00/0
}

cided to use a thick horizontal slice of the basal hypothalamus/ preoptic area to examine the potential influence of the SCN on VLPO neurons.

\section{Materials and Methods}

Horizontal slice preparation. Rats were reared at the animal facility of the Geneva Medical Center and handled according to the regulations of the Swiss Federal Veterinary Office. In brief, young rats (15-21 d) were decapitated, their brains were removed, and a block of the anterior hypothalamus/preoptic area (AH/POA) was obtained (Fig. $1 A$, left inset, lines a-c indicate the approximate location at which the brain block was cut to prepare the horizontal slice). It was then fixed to the stage of a vibroslicer (VT 1000S; Leica Microsystems, Nussloch, Germany), and a single section produced a unique 500 - to $600-\mu \mathrm{m}$-thick horizontal slice having its ventral surface intact (Fig. $1 \mathrm{~A}$ ). The horizontal slice was then transferred to a thermoregulated chamber $\left(32^{\circ} \mathrm{C}\right)$ with its sectioned dorsal surface up. It was then continuously superfused at $4-5 \mathrm{ml} / \mathrm{min}$ with an oxygenated $\left(95 \% \mathrm{O}_{2} / 5 \% \mathrm{CO}_{2}\right.$ ) artificial CSF (ACSF) containing the following (in mM): $130 \mathrm{NaCl}, 5 \mathrm{KCl}, 1.25 \mathrm{KH}_{2} \mathrm{PO}_{4}, 1.3 \mathrm{MgSO}_{4}, 20 \mathrm{NaHCO}_{3}, 10$ glucose, and $2.4 \mathrm{CaCl}_{2}$. Bipolar electrical stimulating electrodes or electrodes allowing for the local pressure application of NMDA were placed in the SCN (Fig. $1 A$ ) previously localized $(n=5)$ by recording a typical SCN field potential (Shibata et al., 1984) in response to contralateral optic nerve stimulation.

Electrophysiology. For extracellular recordings in the loose-attached cell configuration, patch electrodes (5-7 M $\Omega$ ) were filled with ACSF. For whole-cell recordings, the pipettes (7-9 M $\Omega$ ) contained the following solution (in mM): $126 \mathrm{KMeSO}_{4}$, 8 phosphocreatine, $4 \mathrm{KCl}, 5 \mathrm{MgCl}_{2}, 10$ HEPES, $3 \mathrm{Na}_{2}$ ATP, $0.1 \mathrm{GTP}$, and 0.1 BAPTA. In this condition, the reversal potential for chloride is fixed to $-60 \mathrm{mV}$. Current- and voltageclamp recordings were made (between 10:00 A.M. and 5:00 P.M.) using an Axopatch 200 B (Molecular Devices, Union City, CA). The SCN was stimulated electrically through either a bipolar concentric electrode (internal and external diameters, 50 and $100 \mu \mathrm{m}$, respectively) or a bipolar twisted electrode (diameter, $100 \mu \mathrm{m}$ ). Single pulses (intensity, 200-250 


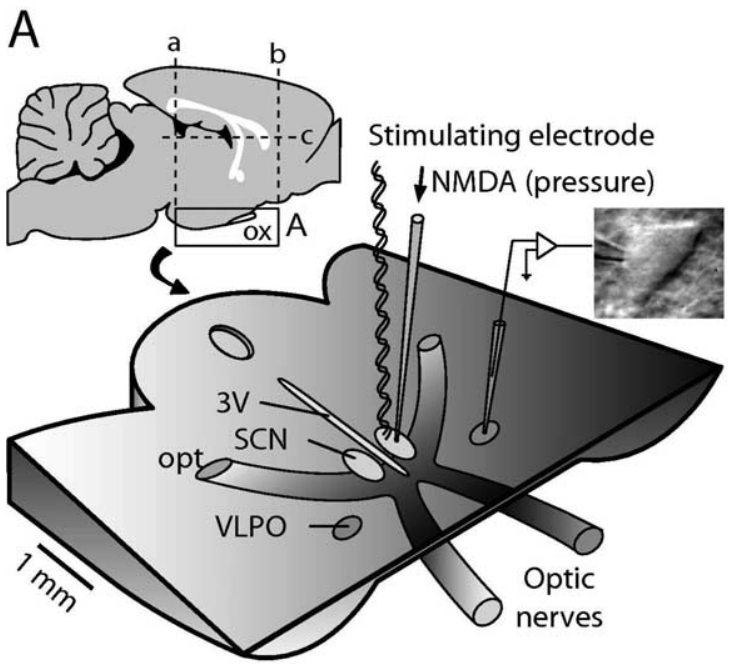

B
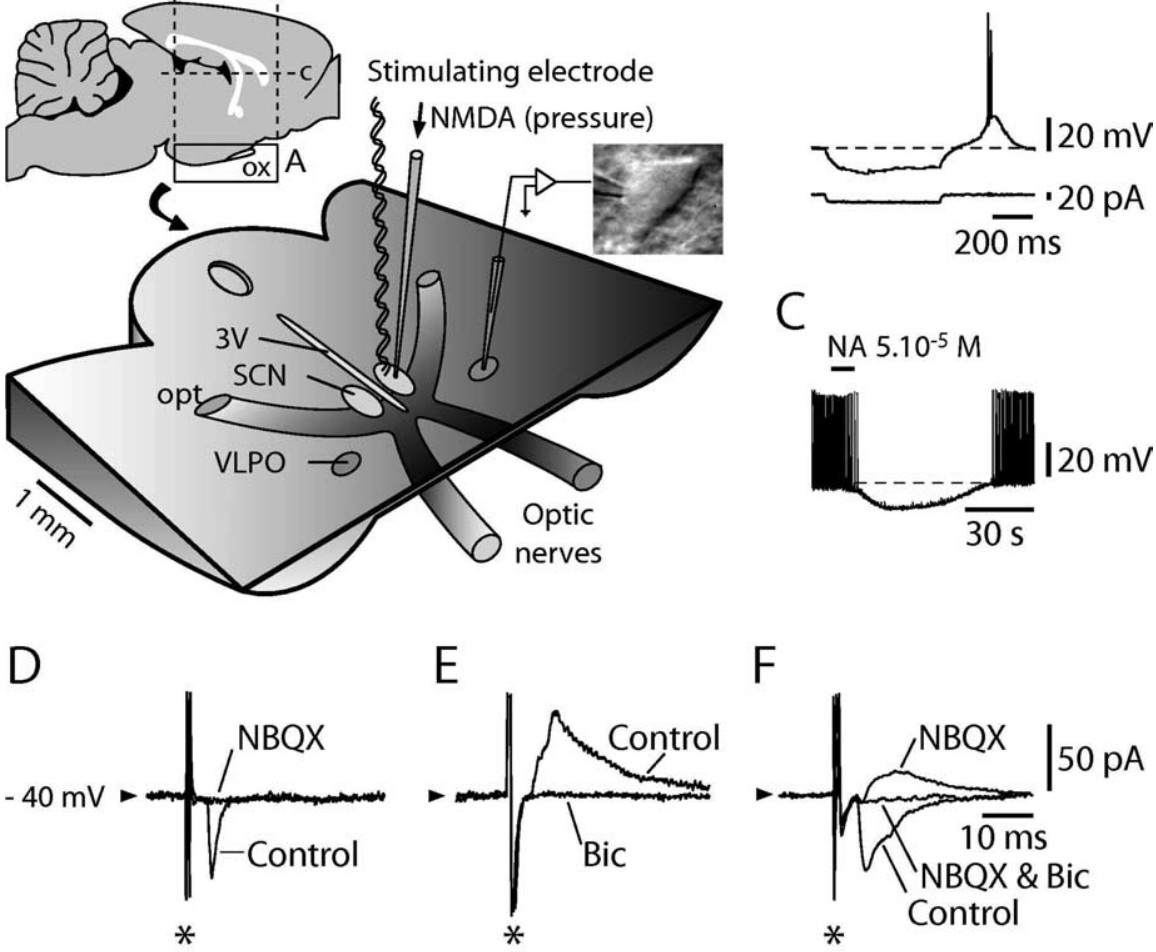

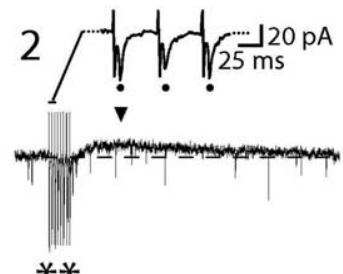

$* *$

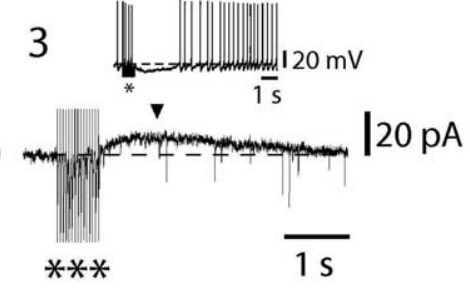

Figure 1. Multiple effects of SCN stimulation on identified sleep-promoting VLPO neurons. $A$, Schematic view of the acute AH/POA horizontal slice preparation. Insets, Sections (lines a - c) performed to prepare the horizontal slice (left inset) and a typical triangular-shaped neuron recorded in the VLPO (right inset). $B, C, A$ typical VLPO neuron recorded in whole-cell configuration in the current-clamp mode displays a low-threshold spike at the end of a hyperpolarizing current step $(\boldsymbol{B})$ and is transiently hyperpolarized and inhibited $(\boldsymbol{C})$ by bath-applied NA. $\boldsymbol{D}-\boldsymbol{F}$, In voltage-clamp mode, SCN single-pulse stimulations $\left({ }^{*} ; 200 \mu A\right)$ evoke NBQX $(25 \mu \mathrm{m})$-sensitive EPSCS $(\boldsymbol{D})$, bicuculline $(20 \mu \mathrm{m})$-sensitive IPSCS $(\boldsymbol{E})$, or a mixture of both $(\boldsymbol{F})$. Traces in $\boldsymbol{D}-\boldsymbol{F}$ are an average of 10 consecutive stimulations. $\mathbf{G}$, Whereas an SCN single-pulse stimulation (200 $\mu \mathrm{A} ; \mathbf{G 1}$, *) evokes a unique PSC (dot in inset), train stimulations of increasing duration $\left(\mathbf{G} 2,{ }^{* *} ; \mathbf{G}{ }^{* * *}\right)$ evoke, in addition to reliable PSCs after each single stimulation $(\mathbf{G 2}$, dots in inset), a long-lasting outward current (G2, G3, arrowheads). As evident when recording the same neuron in current-clamp mode, such a current subtended a long-lasting hyperpolarization (G3, inset). $3 \mathrm{~V}$, Third ventricle; opt, optic tract; $0 \mathrm{x}$, optic chiasm; Bic, bicuculline. $\mu \mathrm{A}$; duration, $0.5-1 \mathrm{~ms}$ ) or train pulses (intensity, mostly between 100 and $200 \mu \mathrm{A}$; duration, 400-750 ms; frequency, 15-20 Hz in most cases and $1-2 \mathrm{~Hz}$ in three cases) were delivered using isolated batteries. Alternatively, SCN stimulation could be performed by local pressure ejection (pressure, 5 psi; duration, 2-10 s) of NMDA at $1 \mathrm{~mm}$. It is noteworthy that responses to NMDA applications had lower amplitudes and slower kinetics than electrical stimulations. This must be the result of the relatively slow diffusion of neurotransmitter agonists through the tissue as well as of a lesser recruitment (compared with electrical stimulation) of neurons within a particular area. Phosphonovaleric acid (APV), bicuculline, (2S)-2-amino-2-[(1S,2S)-2-carboxycycloprop-1-yl]-3(xanth-9-yl) propanoic acid (LY341495), 2,3-dioxo-6-nitro-1,2,3,4-tetrahydrobenzo $[f]$ quinoxaline-7-sulfonamide (NBQX), NMDA, and yohimbine were all obtained from Tocris Bioscience (Bristol, UK) and tetrodotoxin (TTX) from Latoxan (Rosans, France).

\section{Results}

Fast postsynaptic currents and longlasting inhibition evoked by SCN stimulation

Under infrared video microscopy, recordings were obtained (Fig. 1A) from neurons located in the core VLPO, where a cluster of sleep-active neurons were originally identified (Sherin et al., 1996, 1998). Cells were selected as putatively sleep promoting according to previously established criteria (Gallopin et al., 2000). First, they were triangular in shape (Fig. $1 A$, right inset); second, they were inhibited by noradrenaline (NA) (illustrated in Fig. $1 C)$; and finally, they displayed a characteristic low-threshold spike (LTS) at the end of hyperpolarizing current steps when recorded in current-clamp mode (Fig. $1 B)$. Triangular-shaped cells inhibited by NA always displayed an LTS.

As a first step, we tested the effects of single-pulse electrical stimulations applied in the ipsilateral SCN on voltageclamped identified VLPO cells. Postsynaptic currents (PSCs) could be evoked in 18 of 20 cells $(85.7 \%)$, and their pharmacology was characterized in a subset of 10 neurons. In three of 10 cells (30\%), the evoked PSCs were AMPA-dependent EPSCs, as judged by the suppression of the evoked inward currents with NBQX (Fig. $1 D)$. In four of 10 cells $(40 \%)$, they were $\mathrm{GABA}_{\mathrm{A}}$-dependent IPSCs, as judged by the suppression of the evoked outward currents by bicuculline (Fig. $1 E$ ), and in the remaining three cells $(30 \%)$, they were composite EPSCs and IPSCs (Fig. 1F).

Whereas single-pulse stimulations evoked only fast PSCs (Fig. $1 G 1,{ }^{\star}$ ), train stimulations of increasing duration (Fig. $\left.1 G 2,{ }^{* *} ; G 3,{ }^{* *}\right)$ evoked a slow outward current that outlasted the train $(n=3)$ (Fig. 1G2,G3, arrowheads). As evident when switching to current-clamp recordings, such a current subtended a longlasting inhibition (LLI) (Fig. 1G3, inset). As expected for a response resulting from the activation of a pathway, the LLI was abolished in the presence of TTX at $0.5 \mu \mathrm{M}$ ( $n=3$ of 3 ; data not shown).

The long-lasting inhibition depends specifically on the activation of the SCN

That the LLI was indeed the result of a selective activation of the $\mathrm{SCN}$ required a number of control experiments. For that purpose and to maintain stable recordings while changing the place of stimulating electrodes to map the efficient stimulating areas or while switching between electrical and chemical stimulation of the $\mathrm{SCN}$, we opted for extracellular recordings using a looseattached cell configuration. Triangular-shaped multipolar VLPO cells were first identified visually and then tested for their inhibitory response to NA (Fig. $2 A$ ). We then tested whether train 
stimulations at $20 \mathrm{~Hz}$ had an effect similar to the one observed with intracellular recordings. As shown in Figure 2 B1, this was indeed the case, whereas stimulations at a lower frequency ( $2 \mathrm{~Hz} ; n=3$ ) (Fig. 2 B2) had no effect. In addition, train stimulations of increasing amplitudes in the SCN evoked an LLI of increasing duration in these cells (Fig. 2C1-C3).

One first possibility to account for these results could be that current spread from the stimulating electrode directly depolarized the recorded cells, leading to their inactivation. Although such an explanation could already not be reconciled with the results of the TTX experiments reported above, we further excluded this possibility by showing that the LLI was totally but reversibly suppressed in the presence of a high-magnesium $(10 \mathrm{~mm}) /$ lowcalcium (0.1 mM) ACSF known to block synaptic transmission (Fig. 2D1-D3) $(n=$ 6 of 6 ). A final test to exclude this possibility was made by comparing the effects of stimulations performed in the SCN or outside of it, at a location that was closer to the VLPO (Fig. 2E). Whereas SCN train stimulations (Fig. $2 E$, region a) evoked a characteristic LLI ( $n=5$ of 5 ) (Fig. $2 \mathrm{Fa}$ ), the same or even greater amplitude stimulations applied outside the SCN in the anterior hypothalamic area (Fig. $2 E$, region b) had, in contrast, no effect $(n=5$ of 5) (Fig. 2Fb). Moreover, train stimulations of an amplitude similar to or higher than those in the SCN applied to the optic chiasm had no effect $(n=4)$ (Fig. $2 G)$, demonstrating that the LLI was likely not caused by current spread to the optic chiasm. These results, together with those observed in the presence of TTX or synaptic blockade (above), appear to exclude that the effect was caused by direct current spread to VLPO neurons or closely located terminals or to fibers within the optic chiasm. They suggest, in contrast, that the LLI likely depends on the release of a transmitter.

A second possibility could be that rather than stimulating SCN neurons, the stimulus activated fibers of passage. To exclude that possibility, NMDA was applied by pressure ejection in the SCN (Fig. $2 H$ ). As shown in Figure $2 I$ for a typical VLPO neuron inhibited by NA (data not shown), both SCN train stimulation (Fig. 2 I1) and the local application of NMDA at $1 \mathrm{~mm}$ in the SCN (Fig. 2 I2) induced a long-lasting reversible decrease in the spontaneous firing rate. It is noteworthy, however (see Materials and Methods), that the NMDA-induced LLI was weaker, albeit longer lasting, than the one evoked by electrical stimulation of the SCN. The NMDA-evoked inhibitory effect could be observed in 13 of $36(36.1 \%)$ tested neurons, with a change in average firing rate from $7.48 \pm 0.82$ to $5.29 \pm 0.98 \mathrm{~Hz}$ (paired $t$ test; $p<0.001$ ), corresponding to $36.4 \pm 8.7 \%$ inhibition. The firing of the remaining 23 cells was either not affected $(n=22$ of 36 ; nerve; stim, stimulation
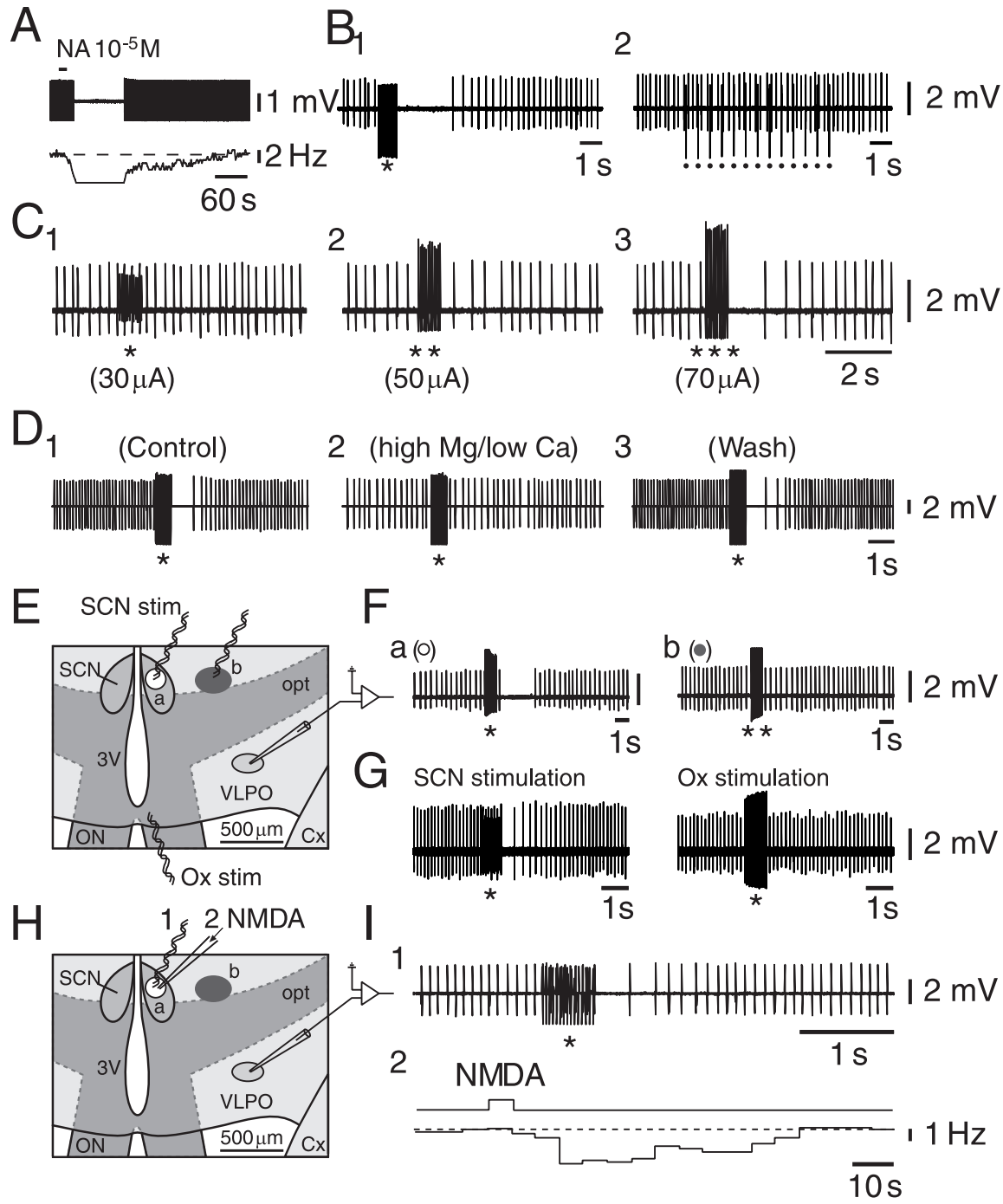

Figure 2. LLI in the firing of identified VLPO neurons depends on SCN stimulation. $A$, Typical NA-induced inhibition of a VLPO neuron recorded in loose-attached cell configuration. $\boldsymbol{B}$, Twenty hertz $\mathrm{SCN}$ train stimulations $\left(\boldsymbol{B}_{1}{ }^{*}\right)$ in contrast with $2 \mathrm{~Hz}$ train stimulations (B2, dots) produce an LLI. C, SCN train stimulations of increasing amplitude $\left[\mathbf{C} 1,30 \mu \mathrm{A}\left({ }^{*}\right) ; \mathbf{C}, 50 \mu \mathrm{A}\left({ }^{* *}\right) ; \mathbf{C}, 70 \mu \mathrm{A}\right.$ $\left.{ }^{* * *}\right)$ ] produce an LLI of increasing duration and strength in an identified VLPO neuron. $\boldsymbol{D}$, The SCN-evoked (*;200 $\mu$ A) LLI (D1) is totally (D2) and reversibly (D3) prevented in presence of a high-magnesium/low-calcium-containing ACSF known to interfere with synaptic transmission. $\boldsymbol{E}$, Two regions (a and b) stimulated successively while recording from the same identified VLPO neurons are shown in a schematic view of a selected region of the AH/POA horizontal slice preparation. $\boldsymbol{F}$, Train stimulation ${ }^{*} ; 150$ $\mu \mathrm{A}$ ) applied outside of the SCN (region b) has no effect on its discharge ( $\boldsymbol{F b}$ ). G, Train stimulations applied in the SCN (left) but not in the optic chiasm (right) produce an LLI. $\boldsymbol{H}$, Electrical and chemical stimulations are applied successively in the SCN (region a) while recording the same identified VLPO neuron. $I$, The same VLPO neuron is inhibited by SCN train stimulation (I1) and by local pressure application of NMDA at $1 \mathrm{~mm}$ within the $\mathrm{SCN}(\mathbf{I 2}) .3 \mathrm{~V}$, Third ventricle; $\mathrm{C}$, cortex; opt, optic tract; $0 \mathrm{x}$, optic chiasm; 0N, optic

$61.1 \%$ of cells) or, only in one case, increased ( $n=1$ of $36 ; 2.8 \%$ of cells). It is noteworthy that bath applications of NMDA at increasing concentrations (between $10^{-7}$ and $10^{-5} \mathrm{M}$ ) had either no effect or an exclusively excitatory effect on VLPO neurons ( $n=7$ of 7 ) (data not shown), indicating that the NMDAinduced LLI was not attributable to diffusion of NMDA toward the VLPO. This contention is supported by the fact that when NMDA was pressure applied in an adjacent region (Fig. $2 \mathrm{H}$, region b), it did not affect VLPO neurons $(n=2)$ (data not shown), which had previously been inhibited after pressure application of NMDA and electrical stimulation in the SCN (Fig. $2 H$, region a).

Finally, it is noteworthy that the LLI was not simply caused by a long-lasting hyperpolarization after fast PSCs, because it per- 


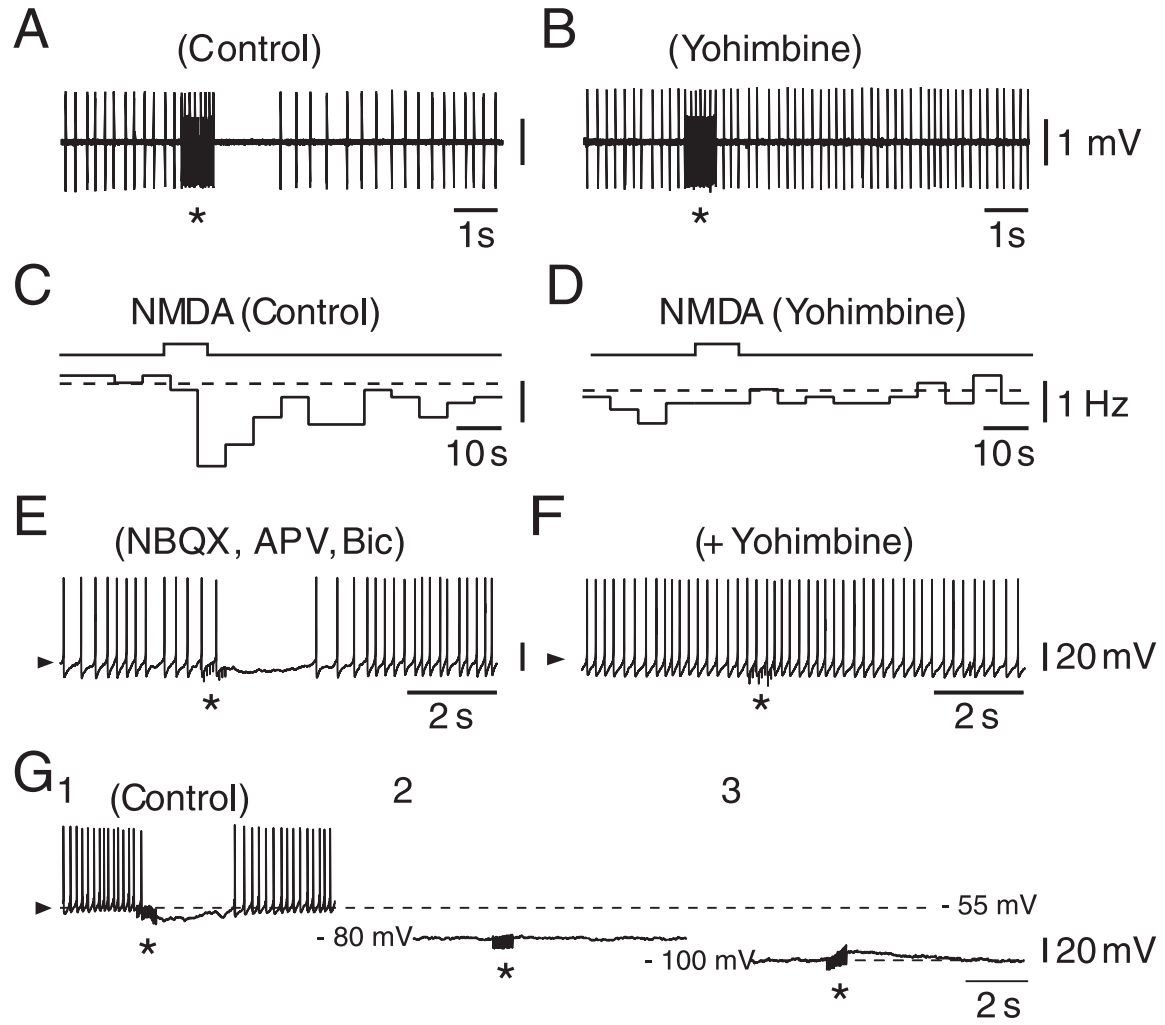

Figure 3. SCN-evoked LLI of identified VLPO neurons depends on the release of noradrenaline. $A, C$, In control conditions, both a train stimulation $\left(\boldsymbol{A}^{*}{ }^{*}\right)$ and selective activation of $S C \mathrm{~N}$ neurons by pressure ejection of NMDA at $1 \mathrm{~mm}$ within the $S C N(C)$ evoke an LLI in identified VLPO neurons. $\boldsymbol{B}, \boldsymbol{D}$, In the presence of yohimbine at $2 \mu \mathrm{M}$, a selective antagonist of $\alpha_{2}$-adrenoreceptors, none of these inhibitory effects (compare $\boldsymbol{A}$ with $\boldsymbol{B}$ and $\boldsymbol{C}$ with $\boldsymbol{D}$ ) can be evoked. $\boldsymbol{E}, \boldsymbol{F}$, In presence of NBQX, APV, and bicuculline (Bic), SCN train stimulation $(* ; 200 \mu \mathrm{A})$ evokes an LLHP in an identified VLPO neuron $(\boldsymbol{E})$, which completely disappears in the presence of yohimbine at $2 \mu \mathrm{M}(\boldsymbol{F}) . \mathbf{G}$, The LLHP observed at the resting membrane potential (G1) in response to SCN train stimulation $\left({ }^{*} ; 200\right.$ $\mu \mathrm{A})$ is annulled at $E_{\mathrm{K}}(\mathbf{G} 2)$, the equilibrium potential for potassium, and is reversed below $E_{\mathrm{K}}(\mathbf{G} \mathbf{3})$.

sisted in the presence of GABAergic [bicuculline (6 of 8) and saclofen (2 of 2) (data not shown)] and glutamatergic blockers [APV and NBQX (5 of 5) or LY341495, a metabotropic receptor antagonist (7 of 8); data not shown].

\section{The long-lasting inhibition depends on the release of noradrenaline}

Given the strength and duration of the LLI, which was reminiscent of that of bath-applied NA, and in view of our recent demonstration in the VLPO of a presynaptic facilitation of NA release by nicotinic agonists (Saint-Mleux et al., 2004), we hypothesized that the LLI could share a similar mechanism. As illustrated in Figure 3, this was indeed the case, because the LLIs evoked electrically (Fig. $3 A$ ) or by NMDA (Fig. $3 C$ ) were completely antagonized [ 8 of 9 cells (Fig. 3B) and 4 of 4 cells (Fig. 3D), respectively] by $2 \mu \mathrm{M}$ yohimbine, a selective $\alpha_{2}$-adrenoreceptor antagonist. Interestingly, however, the persistence of the LLI in presence of dihydro- $\beta$-erythroidine ( $n=4$ of 4$)$, a nonselective nicotinic receptor antagonist, rules out the involvement of nicotinic receptors in this effect. A similar yohimbine-dependent abolition of the LLI was also found when using intracellular recordings $(n=3$ of 3) (Fig. 3, compare E, F). As expected for an $\alpha_{2}$ adrenoreceptor-dependent effect implicating the opening of potassium channels, the LLI was annulled at $E_{\mathrm{K}}$ and could be reversed below it ( $n=4)$ (Fig. 3G1-G3).

\section{Discussion}

The presence of fast GABAergic and glutamatergic PSCs evoked by SCN single-pulse stimulation in VLPO neurons is in accor- dance with a previous study concerning the nature of the outputs of the SCN (Sun et al., 2001) demonstrating GABAergic and glutamatergic inputs from the SCN to the VLPO. Interestingly, however, our results somewhat differ from those of that latter study, in which only unidentified VLPO neurons were tested, and only 30\% of them apparently received an SCN input. Indeed, in the present study, $85.7 \%$ of the VLPO neurons, identified as sleeppromoting according to previously established criteria (Gallopin et al., 2000), received a synaptic input from the SCN in the form of fast PSCs.

In contrast to the fast PSPs obtained with single-pulse stimulation, SCN train stimulation at frequencies compatible with those observed in the firing of SCN neurons (Saeb-Parsy and Dyball, 2003) induced an LLI. The demonstration of this SCN-evoked LLI or long-lasting hyperpolarization (LLHP) in VLPO neurons was entirely new and unexpected and thus necessitated a thorough investigation of possible artifacts. The first and foremost was that the LLI actually resulted from current spread. However, the findings that VLPO neurons were never excited or depolarized by electrical stimuli and that the LLI did not occur in presence of either TTX or high-magnesium/low-calcium ACSF are inconsistent with such a hypothesis. A second possibility would be that the spread of current could directly depolarize various presynaptic elements within the VLPO, including terminals contacting the sleep-promoting neurons (or presynaptic boutons contacting presynaptic terminals that contact VLPO neurons). If, for example, noradrenergic or cholinergic terminals were considered, such a direct presynaptic depolarization would result in the silencing of the VLPO neurons given the strong inhibitory effects of these transmitters (Gallopin et al., 2000; Saint-Mleux et al., 2004). Such an interpretation is, however, again unlikely because cells responding by an LLI to an SCN train stimulation were not affected at all by stimulations (of the same or even greater amplitude) applied outside of the SCN in a region even closer to the VLPO. In addition, the inability to evoke an LLHP in the presence of TTX is also inconsistent with such an interpretation. A third and last possibility would be that electrical stimulation could activate fibers of passage (noradrenergic or cholinergic ones, for example). However, the evidence that a yohimbine-sensitive LLI is evoked only when NMDA is pressure ejected into the SCN but not into its vicinity (even when applied closer to the VLPO) does not support this possibility. Because the LLI is totally suppressed by yohimbine, a selective $\alpha_{2}$-adrenoreceptor antagonist, and because the LLHP reverses at $E_{\mathrm{K}}$, the present results point to a mechanism similar to the one we previously described that shows a presynaptic facilitation of NA release by nicotinic agonists (Saint-Mleux et al., 2004).

In contrast to our previous study with acetylcholine, however, a major difficulty here is to propose a mechanism by which NA release is modulated. Indeed, neither GABA nor glutamate appears to be involved, because the LLI persists in the presence of 
various blockers of these transmitters' receptors. In addition, the persistence of the LLI in the presence of a nicotinic blocker also refutes a role for a presynaptic nicotinic modulation. At that stage, the mechanism involved thus remains a matter of speculation; however, the fact that the LLI (as opposed to short-lasting EPSPs or IPSPs) appears only in presence of train stimulations speaks in favor of the involvement of a peptide. In that context, it is noteworthy that SCN neurons seem to express many different peptides (Moore et al., 2002), which could, directly or indirectly, act on noradrenergic terminals to enable the release of NA. The most prominent among these are arginine vasopressin, vasoactive intestinal polypeptide, and gastrin-releasing peptide. It should finally be mentioned that, even in our reduced preparation, neurons located in the immediate vicinity of the SCN could serve as a relay of the suprachiasmatic input to the VLPO (Leak and Moore, 2001; Deurveilher et al., 2002). Altogether, it was, however, deemed beyond the scope of the present study to identify the transmitter and eventual local pathway possibly involved in the release of NA on VLPO neurons.

Previous studies have shown rat SCN neurons to be more active during the subjective day, when animals are predominantly asleep (Meijer et al., 1997, 1998; Deboer et al., 2003) and when the sleep-promoting VLPO neurons would also be most active (Sherin et al., 1996). This profile of SCN activity in relation to VLPO activity appears to contradict our results, because we show that increased activity in SCN neurons produces an inhibition of VLPO sleep-promoting neurons. However, recent in vitro studies have shown that neurons in the SCN are heterogeneous in their firing with respect to circadian time (Welsh et al., 1995; Herzog et al., 1997; Jagota et al., 2000; Schaap et al., 2003). Moreover, it was recently revealed by in vivo recordings that SCN unit activity changes in parallel with changes in sleep-wake state and independently from circadian phase (Deboer et al., 2003). Indeed, during both phases of the circadian cycle, SCN neurons show a decreased activity during slow-wave sleep with respect to waking and rapid eye movement sleep. Such a negative correlation between SCN neuronal activity and slow-wave activity characteristic of slow-wave sleep would be consistent with an SCNmediated inhibition of VLPO neurons and consequential attenuation of sleep. As a final point, it is important to note that SCN lesions in the rat fail to (Mistlberger et al., 1983; Tobler et al., 1983) or only slightly increase sleep (Abrahamson and Moore, 2006). This result could be attributed to the evidence that the sleep/wake cycle and the maintenance of one or the other state are under the control of at least two mechanisms, one being indeed circadian but the other being homeostatic and independent of SCN integrity. In conclusion, our results suggest that the SCN can influence the sleep-waking cycle, notably by attenuating sleep through the inhibition of VLPO sleep-promoting neurons. It is evident that this pathway is one among a number that link circadian rhythms and the sleep-wake cycle (Deurveilher et al., 2002; Saper et al., 2005).

\section{References}

Abrahamson EE, Moore RY (2006) Lesions of suprachiasmatic nucleus efferents selectively affect rest-activity rhythm. Mol Cell Endocrinol 252:46-56.

Chou TC, Bjorkum AA, Gaus SE, Lu J, Scammell TE, Saper CB (2002) Afferents to the ventrolateral preoptic nucleus. J Neurosci 22:977-990.

Deboer T, Vansteensel MJ, Detari L, Meijer JH (2003) Sleep states alter activity of suprachiasmatic nucleus neurons. Nat Neurosci 6:1086-1090.

Deurveilher S, Burns J, Semba K (2002) Indirect projections from the suprachiasmatic nucleus to the ventrolateral preoptic nucleus: a dual tracttracing study in rat. Eur J Neurosci 16:1195-1213.
Gallopin T, Fort P, Eggermann E, Cauli B, Luppi PH, Rossier J, Audinat E, Mühlethaler M, Serafin M (2000) Identification of sleep-promoting neurons in vitro. Nature 404:992-995.

Gong H, McGinty D, Guzman-Marin R, Chew KT, Stewart D, Szymusiak R (2004) Activation of c-fos in GABAergic neurones in the preoptic area during sleep and in response to sleep deprivation. J Physiol (Lond) 556:935-946.

Herzog ED, Geusz ME, Khalsa SB, Straume M, Block GD (1997) Circadian rhythms in mouse suprachiasmatic nucleus explants on multimicroelectrode plates. Brain Res 757:285-290.

Jagota A, de la Iglesia HO, Schwartz WJ (2000) Morning and evening circadian oscillations in the suprachiasmatic nucleus in vitro. Nat Neurosci 3:372-376.

Jones BE (2005) Mechanisms of sleep-wake states. In: Principles and practice of sleep medicine, Ed 4 (Kryger MH, Roth T, Dement WC, eds), pp 136-153. Philadelphia: Elsevier Saunders.

Kalsbeek A, Buijs RM (2002) Output pathways of the mammalian suprachiasmatic nucleus: coding circadian time by transmitter selection and specific targeting. Cell Tissue Res 309:109-118.

Leak RK, Moore RY (2001) Topographic organization of suprachiasmatic nucleus projection neurons. J Comp Neurol 433:312-334.

Meijer JH, Schapp J, Watanabe K, Albus H (1997) Multiunit activity recordings in the suprachiasmatic nuclei: in vivo versus in vitro models. Brain Res 753:322-327.

Meijer JH, Watanabe K, Schaap J, Albus H, Detari L (1998) Light responsiveness of the suprachiasmatic nucleus: long-term multiunit and singleunit recordings in freely moving rats. J Neurosci 18:9078-9087.

Mistlberger RE (2005) Circadian regulation of sleep in mammals: role of the suprachiasmatic nucleus. Brain Res Brain Res Rev 49:429-454.

Mistlberger RE, Bergmann BM, Waldenar W, Rechtschaffen A (1983) Recovery sleep following sleep deprivation in intact and suprachiasmatic nuclei-lesioned rats. Sleep 6:217-233.

Modirrousta M, Mainville L, Jones BE (2004) Gabaergic neurons with alpha2-adrenergic receptors in basal forebrain and preoptic area express c-Fos during sleep. Neuroscience 129:803-810.

Moore RY, Speh JC, Leak RK (2002) Suprachiasmatic nucleus organization. Cell Tissue Res 309:89-98.

Novak CM, Nunez AA (2000) A sparse projection from the suprachiasmatic nucleus to the sleep active ventrolateral preoptic area in the rat. NeuroReport 11:93-96.

Saeb-Parsy K, Dyball RE (2003) Defined cell groups in the rat suprachiasmatic nucleus have different day/night rhythms of single-unit activity in vivo. J Biol Rhythms 18:26-42.

Saint-Mleux B, Eggermann E, Bisetti A, Bayer L, Machard D, Jones BE, Muhlethaler M, Serafin M (2004) Nicotinic enhancement of the noradrenergic inhibition of sleep-promoting neurons in the ventrolateral preoptic area. J Neurosci 24:63-67.

Saper CB, Scammell TE, Lu J (2005) Hypothalamic regulation of sleep and circadian rhythms. Nature 437:1257-1263.

Schaap J, Albus H, VanderLeest HT, Eilers PH, Detari L, Meijer JH (2003) Heterogeneity of rhythmic suprachiasmatic nucleus neurons: implications for circadian waveform and photoperiodic encoding. Proc Natl Acad Sci USA 100:15994-15999.

Sherin JE, Shiromani PJ, McCarley RW, Saper CB (1996) Activation of ventrolateral preoptic neurons during sleep. Science 271:216-219.

Sherin JE, Elmquist JK, Torrealba F, Saper CB (1998) Innervation of histaminergic tuberomammillary neurons by GABAergic and galaninergic neurons in the ventrolateral preoptic nucleus of the rat. J Neurosci 18:4705-4721.

Shibata S, Oomura Y, Kita H, Liou SY, Ueki S (1984) Field potentials in the suprachiasmatic nucleus of rat hypothalamic slice produced by optic nerve stimulation. Brain Res Bull 12:377-379.

Sun X, Whitefiels S, Rusak B, Semba K (2001) Electrophysiological analysis of suprachiasmatic nucleus projections to the ventrolateral preoptic area in the rat. Eur J Neurosci 14:1257-1274.

Szymusiak R, Alam N, Steininger TL, McGinty D (1998) Sleep-waking discharge patterns of ventrolateral preoptic/anterior hypothalamic neurons in rats. Brain Res 803:178-188.

Tobler I, Borbely AA, Groos G (1983) The effect of sleep deprivation on sleep in rats with suprachiasmatic lesions. Neurosci Lett 42:49-54.

Welsh DK, Logothetis DE, Meister M, Reppert SM (1995) Individual neurons dissociated from rat suprachiasmatic nucleus express independently phased circadian firing rhythms. Neuron 14:697-706. 